\title{
Fast Implementations of the Kalman Bucy Filter for Satellite Data Assimilation
}

\author{
Amir Asif, Senior Member, IEEE
}

\begin{abstract}
We present practical data assimilation algorithms based on the Kalman Bucy filter (KBf) for combining satellite altimetry data with the nonlinear ocean circulation models. Data assimilation in such applications is computationally challenging because of the large dimensions of the state fields. Compared with the direct KBf, our KBf implementations provide computational savings of two orders of the magnitude of the linear dimension of the state field. We run twin experiments by interfacing our data assimilation algorithms with the NLOM, a nonlinear ocean circulation model developed at the Naval Research Laboratory (NRL).
\end{abstract}

\section{INTRODUCTION}

Estimating the state of the ocean fields (for example, the sea surface height and ocean velocity components) is a key issue in physical oceanography. Direct application of the Kalman Bucy filter (KBf) in such data assimilation, [1]-[5], problems is difficult for two reasons. First, the state equations are nonlinear and the resulting $\mathrm{KBf}$ is nonrigorous. Second, the $\mathrm{KBf}$ involves formidable computational requirements in inverting and storing the covariance matrices such that its application is limited to relatively simple oceanographic models.

Main Contributions: The focus of the paper is as follows: 1. To develop a practical implementation of the local KBf, [2], for nonlinear ocean circulation models. While [2] is limited to dynamical models arising from the discretization of linear partial differential equations (pde), we extend the work to nonlinear ocean circulation models such as the Navy layered ocean model (NLOM), [6]. A second difference arises due to the difference in the structure of the state matrices. While the state matrices in [2] are block banded, the state matrices in the NLOM are full matrices. However, the blocks that constitute the state matrices are subblock banded so, the local $\mathrm{KBf}$ is applied at the subblock level to the NLOM.

2. To generalize the local KBf implementation to an $M$ block banded approximation. Reference [2] applies a tridiagonal $(M=1)$ block banded approximation to the inverse of the error covariance matrix. In this paper, we generalize the result to an $M$-block banded approximation and test how different values of $M$ affect the covariance matrix.

3. To apply the KBf based data assimilation to the NLOM and illustrate experimentally the improvement in the ocean prediction resulting from the data assimilation algorithm.

The paper is organized as follows. Section 2 describes the dynamical models used in ocean circulation. Section 3

Manuscript received January 30, 2003, revised March 13, 2003. This work was supported by the Natural Sciences and Engineering Research Council of Canada (NSERC) through grant no. 228415-03.

Amir Asif is with the Department of Computer Science at York University, Toronto, ON M3J 1P3, Canada. (e-mail:asif@cs.yorku.ca). presents our data assimilation algorithms. Twin experiments are included in section 4 . Section 5 concludes the paper.

\section{DYNAMICAL MODELS}

For compactness, the primitive pdes used in the numerical circulation models are expressed as

$$
\frac{\partial \psi}{\partial t}=\mathcal{D} \psi+\mathcal{F}, \quad \mathcal{D}=\sum_{n_{1}, n_{2}, n_{3}}\left(\frac{\partial}{\partial \theta} \frac{\partial}{\partial \phi} a_{n_{1} n_{2} n_{3}}(\theta, \phi)\right)
$$

where $\psi$ represents multiple $(q)$ fields like sea surface height (SSH) $h$, and the latitudinal and longitudinal velocity components $(u, v)$. The operator $\mathcal{D}$ is the nonlinear pde operator, $\theta$ and $\phi$ are the latitudinal and longitudinal coordinates, and $\mathcal{F}$ incorporates the wind forcings. See [6] for details.

In a numerical simulation, the ocean domain constrained by the latitudinal and longitudinal boundaries is discretized into an $(I \times J)$ grid with $L$ layers. A set of finite difference equations obtained by discretizing (1) using the staggered $\mathrm{C}$ grid Leap frog finite difference scheme, computes the ocean circulation fields $h, u$, and $u$ for each layer. We assume that the discretized ocean fields are arranged in a state vector $\Psi$ following some lexicographic order across the multiple fields. In our derivations, a row of each of the discretized field in layer $\ell$ is first mapped into a $(J \times 1)$ random vector and these are stacked one on the top of the other to form a $(q I J \times 1)$ vector, such as,

$$
\Psi^{(\ell)}=[\underbrace{h_{1}^{T}, u_{1}^{T}, v_{1}^{T}}_{\Psi_{1}^{(\ell)}}, \underbrace{h_{2}^{T}, u_{2}^{T}, v_{2}^{T}}_{\Psi_{2}^{(\ell)}}, \ldots, \underbrace{h_{I}^{T}, u_{I}^{T}, v_{I}^{T}}_{\Psi_{I}^{(\ell)}},]^{T}
$$

for $q=3$ and where $h_{i}=\left[h_{i 1}, h_{i 2}, \ldots, h_{i J}\right]^{T}$, for $1 \leq i \leq I$. A similar notation is used for the field vector $u_{i}$ and $v_{i}$. The superscript $\ell$ denotes the number of the layer under consideration, $(1 \leq \ell \leq L)$. The state vector $\Psi$ consists of field vectors $\Psi^{(\ell)}$ for each layer stacked together in the order of the depth. Additional terms that provide coupling between the vertical layers are also included in the dynamical model of (1). The time step is derived from the Courant-FriedrichLewy (CFL) conditions.

It is interesting to note the structure in the covariance matrix $\mathcal{P}$ of the state vector $\Psi$

$$
\begin{aligned}
& \mathcal{P}=\left[\begin{array}{ccc}
P^{(11)} & \cdot & P^{(1 L)} \\
\cdot & \ddots & \cdot \\
P^{(L 1)} & \cdot & P^{(L L)}
\end{array}\right] \\
& \text { with } P^{\left(\ell_{1} \ell_{2}\right)}=\left[\begin{array}{ccc}
P_{11}^{\left(\ell_{1} \ell_{2}\right)} & \cdot & P_{1 I}^{\left(\ell_{1} \ell_{2}\right)} \\
\dot{\ell}_{I 1}^{\left(\ell_{1} \ell_{2}\right)} & \ddots & P_{I I}^{\left(\ell_{1} \ell_{2}\right)}
\end{array}\right]
\end{aligned}
$$


where $P^{\left(\ell_{1} \ell_{2}\right)}$ is a $(q I J \times q I J)$ block that denotes the covariance between field vectors $\Psi^{\left(\ell_{1}\right)}$ and $\Psi^{\left(\ell_{2}\right)}$ for layers $\ell_{1}$ and $\ell_{2}$ of the ocean field. The covariance blocks $P^{\left(\ell_{1} \ell_{2}\right)}$ are further expanded in terms of $(q J \times q J)$ subblocks $P_{i j}^{\left(\ell_{1} \ell_{2}\right)}$ representing the covariance between the $q$ ocean fields of row $i$ in layer $\ell_{1}$ and the corresponding fields of row $j$ in layer $\ell_{2}$. Satellite Measurements: The satellite tracks are curved, so data is available on a few sites along $N$ adjacent rows, $m, \ldots, p$, in the top layer $(\ell=1)$. The measurements $\mathcal{Y}$ are

$$
\underbrace{\left[\begin{array}{c}
\mathcal{Y}_{m}^{(1)}(k+1) \\
\vdots \\
\mathcal{Y}_{p}^{(1)}(k+1)
\end{array}\right]}_{\mathcal{Y}(k+1)}=\underbrace{\left[\begin{array}{lll}
\Theta(k+1) & \underline{0} & 0
\end{array}\right]}_{\mathcal{H}(k+1)} \Psi(k+1)+\xi
$$

with

$$
\Theta(k+1)=\left[\begin{array}{ccccccccccc}
\underline{0} & \cdot & \underline{0} & \theta_{m} & \underline{0} & \cdot & \underline{0} & \underline{0} & \underline{0} & \cdot & \underline{0} \\
\cdot & \cdot & \cdot & \cdot & \cdot & \cdot & \cdot & \cdot & \cdot & \cdot & \cdot \\
\underline{0} & \cdot & \underline{0} & \underline{0} & \underline{0} & \cdot & \underline{0} & \theta_{p} & \underline{0} & \cdot & \underline{0}
\end{array}\right]
$$

For a total of $L$-vertical layers, the observation matrix $\mathcal{H}(k+1)$ is of dimensions $(q N J \times q L I J)$ and is highly sparse. The constituent blocks $\theta_{i}$ 's of $\Theta$ are also sparse since the satellite provides data only on the SSH, $h$. The velocity components $(u, v)$ are not observed. The variable $\xi$ in (5) represents the measurement noise, assumed Gaussian and $k$ is the time index.

\section{EXTENDED KBF}

To apply the $\mathrm{KBf}$ for data assimilation, we propose the extended filtering approach where a real-time linear approximation of the nonlinear dynamic model (1) is used as the state equation in the KBf. The linearized state model is obtained by approximating (1) with the shallow water model

$$
\begin{aligned}
\frac{\partial h^{(\ell)}}{\partial t}+\frac{H^{(\ell)}}{a \cos \theta}\left[\frac{\partial u^{(\ell)}}{\partial \phi}+\frac{\partial\left(v^{(\ell)} \cos \theta\right)}{\partial \theta}\right] & =0 \quad(7) \\
\frac{\partial u^{(\ell)}}{\partial t}-k_{1} v^{(\ell)}+\sum_{\tau=1}^{L} \frac{h^{(\ell)} G^{(\ell \tau)}}{a \cos \theta} \frac{\partial\left(h^{(\tau)}-H^{(\tau)}\right)}{\partial \phi} & =F_{\phi}(8) \\
\frac{\partial v^{(\ell)}}{\partial t}+k_{1} u^{(\ell)}+\sum_{\tau=1}^{L} \frac{h^{(\ell)} G^{(\ell \tau)}}{a} \frac{\partial\left(h^{(\tau)}-H^{(\tau)}\right)}{\partial \theta} & =F_{\theta}(9)
\end{aligned}
$$

where $k_{1}$ is the Coriolis constant given by $2 \Omega \sin \theta, \Omega$ being the Coriolis parameter. The term $H^{(\ell)}$ denotes the thickness of layer $\ell,\left(F_{\phi}, F_{\theta}\right)$ are the longitudinal and latitudinal components of the forcing term $F$, and $G^{(\ell \tau)}$ is the reduced gravity parameter. Discretizing (7)-(9) with the staggered C-grid Leap Frog finite difference scheme, followed by a real-time Taylor approximation of the nonlinear terms, gives the state equation

$$
\Psi(k+1)=\mathcal{A}(k) \Psi(k)+\mathcal{C}(k) W(k) .
$$

where the state matrices $\mathcal{A}$ and $\mathcal{C}$ are full matrices that are partitioned in terms of the time varying blocks, $\mathcal{A}=\left\{A^{\left(\ell_{1} \ell_{2}\right)}\right\}$ and $\mathcal{C}=\left\{C^{\left(\ell_{1} \ell_{2}\right)}\right\}$, for $\left(1 \leq \ell_{1}, \ell_{2} \leq L\right)$. The blocks $A^{\left(\ell_{1} \ell_{2}\right)}$ and $C^{\left(\ell_{1} \ell_{2}\right)}$ have the same dimensions as the covariance blocks $P^{\left(\ell_{1} \ell_{2}\right)}$ but are highly structured with $A^{\left(\ell_{1} \ell_{2}\right)}$ being $M_{1}$ block banded and $B^{\left(\ell_{1} \ell_{2}\right)}$ being $M_{2}$-block banded. The banded structure arises from the local field interactions resulting from the discretization of the pdes (7)-(9). We emphasize that the linear shallow water approximation, (10), is only used to update the forecast error covariance matrix in the KBf. The nonlinear primitive equations (1) predict the model state.

Data Assimilation Algorithm: Our data assimilation algorithm is an extension of [2] applied to a 3D nonlinear multilayered ocean circulation model with two major differences. First, [2] is limited to a tridiagonal block banded approximation to the inverse of the error covariance matrix. In this paper, we generalize to an $M$-block banded approximation based on theorem 1, [7]. Second, [2] applies the tridiagonal block banded approximation directly to the inverse of the error covariance matrix. In order to exploit the structure of the state matrices $\mathcal{A}$ and $\mathcal{C}$ in (10) to the full extent, we apply the $M$ block banded approximation to the inverse of the block $P^{\left(\ell_{1} \ell_{2}\right)}$ rather than $\mathcal{P}$ itself, which is treated as a full matrix.

Theorem 1: Given blocks $\left\{P_{i j}^{\left(\ell_{1} \ell_{2}\right)}\right\}$ on the first $M$-subblock diagonals of $P^{\left(\ell_{1} \ell_{2}\right)}=\left(B^{\left(\ell_{1} \ell_{2}\right)}\right)^{-1}, B^{\left(\ell_{1} \ell_{2}\right)}$ being $M$ subblock banded, the subblocks outside the $M$-subblock diagonals in $P^{\left(\ell_{1} \ell_{2}\right)}$ are

$$
\begin{aligned}
P_{i j}^{\left(\ell_{1} \ell_{2}\right)}= & {\left[P_{i i+1}^{\left(\ell_{1} \ell_{2}\right)} \ldots P_{i i+M}^{\left(\ell_{1} \ell_{2}\right)}\right] \cdot } \\
& {\left[\begin{array}{ccc}
P_{i+1 i+1}^{\left(\ell_{1} \ell_{2}\right)} & \cdot & P_{i+1 i+M}^{\left(\ell_{1} \ell_{2}\right)} \\
\cdot & \ddots & \vdots \\
P_{i+M i+1}^{\left(\ell_{1} \ell_{2}\right)} & \cdot & P_{i+M i+M}^{\left(\ell_{1} \dot{\ell}_{2}\right)}
\end{array}\right]^{-1}\left[\begin{array}{c}
P_{i+1 j}^{\left(\ell_{1} \ell_{2}\right)} \\
\vdots \\
P_{i+M j}^{\left(\ell_{1} \ell_{2}\right)}
\end{array}\right] }
\end{aligned}
$$

for $1 \leq i<(I-M)$ and $(i+M)<j \leq I$.

Below we outline the data assimilation algorithm by simplifying the $\mathrm{KBf}$ at the block and subblock levels.

Local KBf: Initial conditions: $\widehat{\Psi}(0 \mid 0), \mathcal{P}(0 \mid 0)$, state and observation covariance $(\mathcal{Q}, \mathcal{R})$ at $k=0$.

Step 1: Update the field $\widehat{\Psi}(k+1 \mid k)$ using the NLOM, (1).

Step 2: Update the a priori error covariance matrix. In terms of the blocks of $\mathcal{A}=\left\{A^{\left(\ell_{1} \ell_{2}\right)}\right\}, \mathcal{C}=\left\{C^{\left(\ell_{1} \ell_{2}\right)}\right\}, \mathcal{P}=\left\{P^{\left(\ell_{1} \ell_{2}\right)}\right\}$, and $\mathcal{Q}=\left\{Q^{\left(\ell_{1} \ell_{2}\right)}\right\}$, the error covariance matrix is given by

$$
\begin{aligned}
& P^{\left(\ell_{1} \ell_{2}\right)}(k+1 \mid k)= \\
& \sum_{\tau_{2}=1}^{L}\left(\sum_{\tau_{1}=1}^{L} A^{\left(\ell_{1} \tau_{1}\right)}(k) P^{\left(\tau_{1} \tau_{2}\right)}(k \mid k)\right)\left(A^{\left(\ell_{2} \tau_{2}\right)}(k)\right)^{T} \\
& +\sum_{\tau_{2}=1}^{L}\left(\sum_{\tau_{1}=1}^{L} C^{\left(\ell_{1} \tau_{1}\right)}(k) Q^{\left(\tau_{1} \tau_{2}\right)}(k)\right)\left(C^{\left(\ell_{2} \tau_{2}\right)}(k)\right)^{T},
\end{aligned}
$$

for $\left(1 \leq \ell_{1}, \ell_{2} \leq L\right)$. Eq. (12) includes sum of several product terms of the form, $A^{(* *)} P^{(* *)}(k \mid k)\left(A^{(* *)}\right)_{;}^{T}$ each is expressed in terms of the constituent subblocks as

$$
\begin{aligned}
& {\left[A_{1}^{(*)} P_{i-1 j-1}^{(* *)}+A_{2}^{(* *)} P_{i j-1}^{(* *)}+A_{3}^{(* *)} P_{i+1 j-1}^{(* *)}\right]\left(A_{1}^{(* *)}\right)^{T} } \\
+ & {\left[A_{1}^{(* *)} P_{i-1 j}^{(* *)}+A_{2}^{(*)} P_{i j}^{(* *)}+A_{3}^{(* *)} P_{i+1 j}^{(* *)}\right]\left(A_{2}^{(*)}\right)^{T} } \\
+ & {\left[A_{1}^{(* *)} P_{i-1 j+1}^{(* *)}+A_{2}^{(* *)} P_{i j+1}^{(* *)}+A_{3}^{(* *)} P_{i+1 j+1}^{(* *)}\right]\left(A_{3}^{(* *)}\right)^{T} . }
\end{aligned}
$$

where the block $A^{\left(\ell_{1} \ell_{2}\right)}$ is assumed to be tridiagonal subblock banded $^{1},\left(M_{1}=1\right)$, with subblocks $A_{1}^{\left(\ell_{1} \ell_{2}\right)}, A_{2}^{\left(\ell_{1} \ell_{2}\right)}$, and $A_{3}^{\left(\ell_{1} \ell_{2}\right)}$ on its lower, main, and upper subblock diagonals.

\footnotetext{
${ }^{1}$ The discussion presented here is generalizable to the $M_{1}$-subblock banded case for the state matrix $\mathcal{A}$.
} 
The $M$-subblock banded approximation on block $P^{\left(\ell_{1} \ell_{2}\right)}(k+$ $1 \mid k)$ implies that we update only the main and the first $M$ upper subblock diagonals in each such block. An update of $P_{i j}^{\left(\ell_{1} \ell_{2}\right)}(k+1 \mid k),|j-i| \leq M$, requires subblocks

$$
P_{i j}^{\left(\ell_{1} \ell_{2}\right)}(k \mid k), \quad \text { for }|j-i| \leq(M+2)
$$

from the last iteration of the KBf. Of the required subblocks $P_{i j}^{\left(\ell_{1} \ell_{2}\right)}(k \mid k)$ in (13), the subblocks on the first $M$ subblock diagonals are obtained from the previous iteration of the KBf. The remaining subblocks on diagonals $(M+1)$ and $(M+$ 2 ) are computed from the first $M$-subblock diagonals using theorem 1. A similar procedure is used to approximate $Q^{\left(\ell_{1} \ell_{2}\right)}$. Step 3: Compute the Kalman gain expressed as

$$
\begin{aligned}
& K^{(\ell)}(k+1)=P^{(\ell 1)}(k+1 \mid k) \Theta^{T}(k+1) . \\
& \quad\left[\Theta(k+1) P^{(11)}(k+1 \mid k) \Theta^{T}(k+1)+R^{(11)}(k+1)\right]^{-1}
\end{aligned}
$$

where $K^{(\ell)}$ is the $(q I J \times q N J)$ Kalman gain for layer $\ell$, $(1 \leq \ell \leq L)$. At the subblock level, (14) is reduced to

$$
\begin{aligned}
& K_{i}^{(\ell)}(k+1)=\left[\begin{array}{ccc}
P_{1 m}^{(\ell 1)} \theta_{m}^{T} & \ldots & P_{1 p}^{(\ell 1)} \theta_{p}^{T}
\end{array}\right] . \\
& \left.\qquad \begin{array}{ccc}
\left(\theta_{m} P_{m m}^{(11)} \theta_{m}^{T}+R_{m m}^{(11)}\right) & \cdot & \theta_{m} P_{m p}^{(11)} \theta_{p}^{T} \\
\theta_{p} P_{p m}^{(11)} \theta_{m}^{T} & \ddots & (15) \\
& \cdot & \left(\theta_{p} P_{p p}^{(11)} \theta_{p}^{T}+R_{m m}^{(11)}\right)
\end{array}\right]
\end{aligned}
$$

where $K_{i}^{(\ell)}(k+1)$ is a $(q J \times q N J)$ subblock that represents the Kalman gain for row $i$ of layer $\ell,(1 \leq i \leq I)$ and $(1 \leq \ell \leq L)$. Updating the Kalman gain $K_{i}^{(\ell)}(k+1)$ requires a few subblocks $P_{i j}^{\left(\ell_{1} \ell_{2}\right)}$ from outside the $M$-subblock diagonals in block $P^{\left(\ell_{1} \ell_{2}\right)}$. These are computed from the subblocks within the $M$-subblock diagonal of $P^{\left(\ell_{1} \ell_{2}\right)}$ using theorem 1 . Step 4: Compute the predicted estimate from the measurements. At the block level,

$$
\begin{aligned}
\widehat{\Psi}^{(\ell)}(k+1 \mid k+1) & =\widehat{\Psi}^{(\ell)}(k+1 \mid k)+ \\
K^{(\ell)}(k+1) & {\left[\mathcal{Y}^{(1)}(k+1)-\Theta(k+1) \widehat{\Psi}_{m}^{(1)}(k+1 \mid k)\right], }
\end{aligned}
$$

for $(1 \leq \ell \leq L)$. Expanding further to the subblock level gives

$$
\begin{aligned}
\widehat{\Psi}_{i}^{(\ell)}(k+1 \mid k+1) & =\widehat{\Psi}_{i}^{(\ell)}(k+1 \mid k)+ \\
K_{i}^{(\ell)}(k+1) & {\left[\begin{array}{c}
\mathcal{Y}_{m}^{(1)}(k+1)-\theta_{m} \widehat{\Psi}_{m}^{(1)}(k+1 \mid k) \\
\vdots \\
\mathcal{Y}_{p}^{(1)}(k+1)-\theta_{p} \widehat{\Psi}_{p}^{(1)}(k+1 \mid k)
\end{array}\right], }
\end{aligned}
$$

for $(1 \leq i \leq I)$ and $(1 \leq \ell \leq L)$. The ocean field $\Psi$ is updated one row at a time.

Step 5: Compute the a posteriori covariance matrix using

$$
\begin{array}{r}
P^{\left(\ell_{1} \ell_{2}\right)}(k+1 \mid k+1)=P^{\left(\ell_{1} \ell_{2}\right)}(k+1 \mid k)- \\
K^{\left(\ell_{1}\right)}(k+1) \Theta(k+1) P^{\left(1 \ell_{2}\right)}(k+1 \mid k)
\end{array}
$$

for $\left(1 \leq \ell_{1}, \ell_{2} \leq L\right)$. Simplifying to the subblock level gives

$$
\begin{array}{r}
P_{i j}^{\left(\ell_{1} \ell_{2}\right)}(k+1 \mid k+1)=P_{i j}^{\left(\ell_{1} \ell_{2}\right)}(k+1 \mid k)- \\
K_{1}^{\left(\ell_{1}\right)}(k+1)\left[\begin{array}{c}
\theta_{m} P_{m j}^{\left(1 \ell_{2}\right)}(k+1 \mid k) \\
\vdots \\
\theta_{p} P_{p j}^{\left(1 \ell_{2}\right)}(k+1 \mid k)
\end{array}\right]
\end{array}
$$

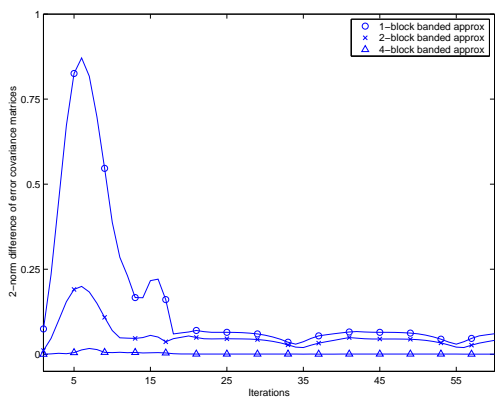

Fig. 1. Comparison of the error covariance matrix from the direct $\mathrm{KBf}$ versus the error covariance matrix approximated by the $M$-block banded approximation in the local KBf.

for $(1 \leq i, j \leq I),|j-i| \leq M$, and $\left(1 \leq \ell_{1}, \ell_{2} \leq L\right)$. Eq. (19) is updated for $P_{i j}^{\left(\ell_{1} \ell_{2}\right)}(k+1 \mid k+1)$ that lie within the first $M$ subblock diagonals. A few subblocks $P_{i j}^{\left(\ell_{1} \ell_{2}\right)}(k+1 \mid k)$ in (19) lie outside the first $M$ subblock diagonals and are computed from the $M$ subblock diagonal blocks using theorem 1.

Step 6: Increment $k$ by 1 and go back to step 1 .

\section{TWIN EXPERIMENT}

To test our data assimilation scheme, an equatorial channel of the Pacific ocean is simulated. The region in consideration lies within the latitudes of $\pm 20^{\circ}$. The longitude varies from $306^{\circ}$ to $374^{\circ}$. Twin experiments on a $1 / 4^{\circ}, 2 \frac{1}{2}$ hydrodynamic NLOM with analytical winds are performed. The dimension of the state vector is roughly of $\mathrm{O}\left(10^{6}\right)$. The wind forcings $\left(F_{\phi}, F_{\theta}\right)$ in (7)-(9) are assumed analytical.

Deterministic setup: runs the NLOM with analytical winds to propagate the deterministic components. Such theoretical estimates are often used by oceanographers to model ocean circulation in various regions of the ocean.

Real world setup: extends the deterministic setup by adding White Gaussian noise with a SNR of $12 \mathrm{~dB}$ to the wind forcings $\left(F_{\phi}, F_{\theta}\right)$. The noise defines the state covariance $\mathcal{Q}$. The two simulations described above are run for a simulated period of 6 months.

Data Assimilation: The output of the real world setup is assimilated as pseudo data, (5), into the deterministic setup to estimate the results of the real world. Gaussian noise of $10 \mathrm{~dB}$ is added to the altimetry observations, $h$. The observation covariance $\mathcal{R}$ is defined by the added observation noise.

Results: To test the data assimilation algorithm, we run two sets of experiments. In experiment 1 , we run a reduced state model described in [7]. The dimensions of the state vector $\Psi$ is 100 , so direct implementation of the $\mathrm{KBf}$ is feasible. Our goal is to test how the value of $M$ in the subblock banded approximation of $P^{\left(\ell_{1} \ell_{2}\right)}$ affects the covariance matrix $\mathcal{P}$. In fig. 1, we show the 2-norm difference between the full error covariance matrix from the direct $\mathrm{KBf}$ and the approximated error covariance matrix based on the $M$-subblock banded approximation to the inverse of the covariance block $P^{\left(\ell_{1} \ell_{2}\right)}$. Fig. 1 illustrates that the $M$-subblock banded approximation is a fairly reasonable approximation improving in accuracy as the value of $M$ is increased. In our data assimilation experiment, we use a pentadiagonal subblock approximation $(M=2)$ 

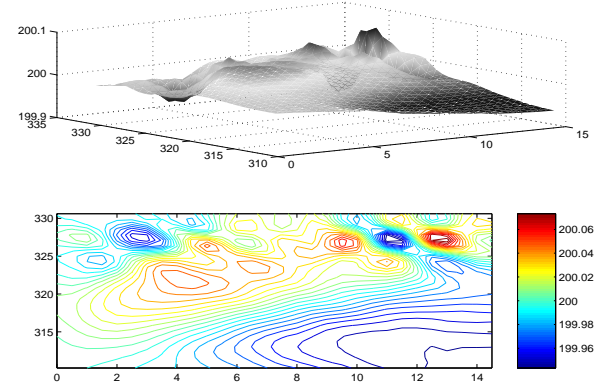

Fig. 2. Mesh and contour plots of the sea surface height (SSH) in meters for the top layer from the real world setup. The scale is shown in colour on the right of the contour plot.
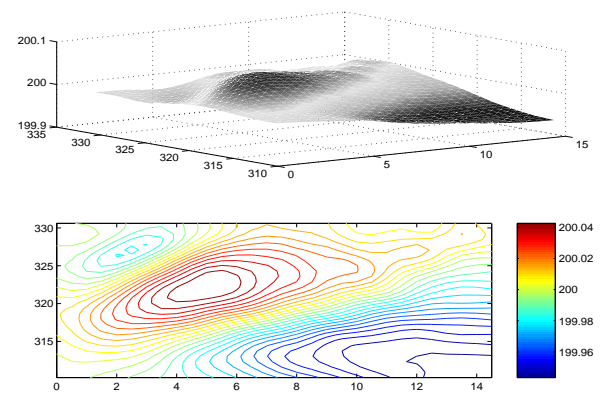

Fig. 3. Mesh and contour plots of the SSH of the top layer from the deterministic setup. The plots do not capture the fi ner details like the eddies, the troughs, and the crests of fig. 2.

in the local KBf. Experiment 2 is used to assimilate the satellite data in the NLOM. Figs. 2, 3, and 4 show estimates of the sea surface height ( $\mathrm{SSH}$ ) for the top layer from the three setups described earlier, at day 36 after three satellite repeat cycles. Both mesh and contour plots are shown. A visual comparison of the figures illustrates that the SSH image associated with the satellite scanned sparse data is a better estimate of the actual ocean state condition, in our case, the real world run, than the field predicted with no data assimilation. The latter is a low pass version of the actual SSH. The data assimilated SSH incorporates the finer details like the eddies as, for example, the valleys and the peaks in fig. 2 are well reproduced in fig. 3 but are smoothed out in fig. 4. In fig. 5, a quantitative comparison based on the mean square error (MSE) is performed. The relative MSE (RMSE) plotted in fig. 5, is defined as the ratio of the MSE of the data assimilated run to the MSE of the deterministic run. The region below ordinate $(y=1)$ represents improvement made by the data assimilated run. With time, the RMSE's for both layers drop suggesting improvements with data assimilation. The improvement is higher, roughly $25 \%$ after 36 days, for the upper layer for which altimetry data is available. No data is assimilated for layer 2, yet we see an improvement of about $10 \%$. Our KBf implementation successfully correlates the two layers and projects the surface information into the lower layer.
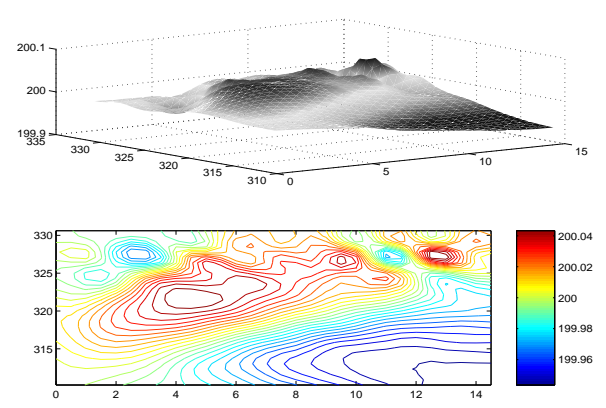

Fig. 4. Mesh and contour plots of the SSH of the top layer from the data assimilation setup. These images illustrate that the data assimilated SSH's are a better estimate of the real world run, fi g. 2.

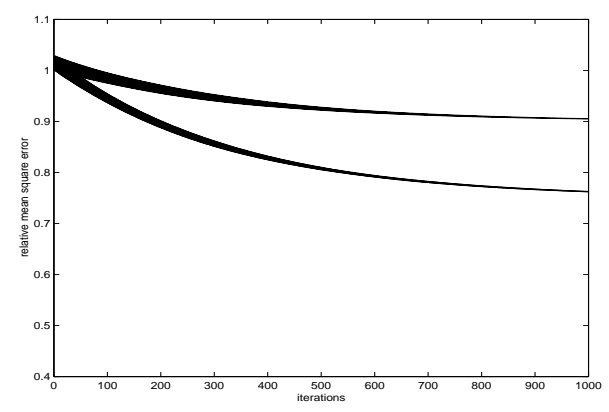

Fig. 5. Relative mean square error (RMSE) of the SSH of the top and the lower layer. The lower curve is the RMSE for the surface layer while the upper curve is the RMSE for the second lower layer.

\section{SUMMARY}

Assimilation of the altimetry data has been investigated for the NLOM. We use a KBf implementation that couples dynamic linearization with an $M$-block banded approximation to the inverse of the error covariance matrix. Twin experiments on a $1 / 2^{\circ}, 2 \frac{1}{2}$ layer hydrodynamic NLOM show significant improvement in the prediction of ocean fields. Although we assimilate data for only the upper layer, reconstructions of all layers are improved. The KBf implementation successfully projects the surface information into the subsurface layers.

\section{REFERENCES}

[1] T. M. Chin, A. J. Mariano, and E. P. Chassignet, "Spatial Regression with Markov Random Fields for Kalman Filter Approximation in Least Squares Solution of Oceanic Data Assimilation Problems," Journal of Geophysical Research, vol. 104, pp. 7991-8014, 1999.

[2] A. Asif and J. M. F. Moura, "Data Assimilation in Large Time Varying Multidimensional Fields," IEEE Transactions on Image Processing, vol. 8, No. 11, Nov. 1999.

[3] D. T. Pham, J. Verron, and M. .C. Roubaud, "A Singular Extended Kalman Filter for Data Assimilation in Oceanography," Journal of Marine Systems, vol. 16, pp. 323-340, 1998.

[4] R. N. Miller and M. A. Cane, "Tropical Data Assimilation: Theoretical Concepts," in Modern Approaches to Data Assimilation in Ocean Modeling, P. M. Rizzoli, Ed., NY: Elsevier, pp. 207 - 233, 1996.

[5] I. Fukumori and P. Malanotte-Rizzoli, "An approximate Kalman fi lter for ocean data assimilation: an example with an idealized Gulf Stream Model," J. of Geophysical Research, vol. 100, pp. 6777-6793, 1995.

[6] D. R. Moore and A. J. Wallcraft, "Formulation of the NRL Layered Ocean Model in Spherical Coordinates," Tech. Rep. CR 7323-96-0005, Nav. Res. Lab., Stennis Space Center, MS, 1995.

[7] A. Asif and J. M. F. Moura, "Inversion of Block Matrices with $L$-Block Banded Inverse," Tech. Rep. Dept. Comp. Sc., York Univ., Toronto, ON, Dec. 2002, submitted for publication. 